\title{
Research of contour extraction for mountain tunnel entrance images
}

\author{
Liu Minjia ${ }^{1,2}$, Lin Jie ${ }^{1,2}$, Yuan Yuyu ${ }^{1,2}$ \\ ${ }^{1}$ School of Computer Science and Technology, Beijing University of Posts and Telecommunications, Beijing 100876, China \\ ${ }^{2}$ Key Laboratory of Trustworthy Distributed Computing and Services, Ministry of Education, Beijing University of Posts and \\ Telecommunications, Beijing 100876, China \\ liuminjiaofbupt@163.com
}

\begin{abstract}
Unmanned aerial vehicle (UAV) needs unmanned ground vehicle (UGV) searching the areas, such as tunnel inside the mountain. The technology of UGV extraction image features of mountain tunnel portal is very important. This paper implies embedded hardware platform to realize the UGV scanning environment image contour feature extraction. A new digital image processing algorithm with MATLAB is expressed for miniaturization real-time image contour feature extraction procedure. The processing speed is fast enough for real-time search task. Otsu method is implied to determine the threshold value, combining with Sobel operator, Prewitt operator and Canny operator to verify that Canny operator has the best effect in images contour extraction. The contour is precision with small amount of calculation.

Index Terms - UAV, cooperative search, wireless communication, image feature extraction, 3G SIM card networking
\end{abstract}

\section{Introduction}

General unmanned control system mainly consists of air navigation UAV and unmanned ground vehicles. When search the unknown environment with relatively high complexity, only UAV and UGV can't completely cover the whole searching task, such as the objectives to judge whether inductive interest mission objectives hiding inside the mountain tunnel. UAV can't cover and scan the inside tunnel without crashing onto the wall. Even a very experienced remote control operator won't prevent UAV from knocking onto the board inside a narrow tunnel. Another kind of situation is that when the ground is extremely rugged with fallen trees and large stones, UGV can be replaced by UAV to search the environment. This kind of complex environment needs UAV and UGV performing the search tasks with coordination.

\section{Complex search tasks need UAV and UGV working with coordination}

Unmanned aerial vehicle (UAV) and unmanned ground vehicle (UGV) can be remote controlled by the remote ground station operators using the computer servers and display screen [1]. When the human pilots and drivers can't risk their lives to search the dangerous environment, such as fire disaster in the forest, the harsh environment covered with too thick snow, the wetland swamp that threats human lives, as well as danger zone that is covered by barbed wire, ditch or gas bombs. UAV and UGV can complete the search task without losing human lives.

With the research on computer autonomous system, UAV and UGV can work without the remote controller. Using the self-organization path planning method for UAV path planning, in accordance with the most efficient search the entire task environment and complete the search task.

\section{The kind of the UAV and UGV in the complex task}

UAV with fixed wing aircraft will execute the search task well when the environment is not complicated, and the image sensors on the UAV perform high accuracy with low failure, especially in a short period of time to quickly determine the unknown environment with high efficiency. However, if the search conditions are complex, and the UAV image sensor needs continuous ten images to confirm the target, fixed wing aircraft are too fast with a large turning radius, and they are not fit. Not until this kind of UAV confirms the tasks in the complex environment will the UAV fly away from the area. With the large turning radius it's not easy to fly around this area again.

A simple solution is to use a helicopter or four axis aircraft instead of fixed wing aircraft. If the search task does not require very strict searching time, the low speed of the helicopter or four axis aircraft is not a big disadvantage. The helicopter can hover to get a full search for regional environment that needs repeated scanning with enough time to confirm the suspected targets.

Along with the third generation mobile communication being more popular, the mature $3 \mathrm{G}$ communication can provide high broadband networking resource.

While searching for the internal of mountain tunnel, UAV will easily be damaged by crashing onto the wall. Through releasing the UGV in the tunnel entrance, the UGV can take over the search task. The general choice of capable hovering helicopter will make the UGV landing easier.

The complex environment requires the type of UGV to be the off-road vehicle that can deal with rugged condition. Because the UGV landing may be not perfect, and the off-road vehicle will drive in complex environment, the memory metal alloy with light weight and steady shape is the best choice to make the UGV. If funds are limited, the polycarbonate material and carbon fiber material also can be a good choice to make the UGV.

\section{UGV landing near the mountain tunnel entrance}

When executing the search task in complex environment, the potential target sites hide among the trees and UGV can't drive. Then UAV will land the UGV near the potential target 
sites. UGV opens the parachute safely with a distance of 5 meters high from the ground by airborne sonar detectors. The choose of sonar detectors is because the existent of four level wind is common in the searching tasks, the low landing height for UGV opening the parachute can avoid the UAV landing too far away from the tunnel entrance. Design of the UAV can bear the bad weather in the tasks and can drive in complex pavement.

In an adverse environment, UAV find the entrance region of mountain tunnel mainly through the images of airborne image sensors. The remote control personnel in the remote ground stations will judge the entrance with the received images by naked eyes.

\section{UGV scans to confirm the mountain tunnel entrance around the landing site}

When the UGV lands around the mountain tunnel entrance, it will scan the surrounding environment to determine the mountain tunnel entrance. This is usually connected to remote ground control station server through the $3 \mathrm{G}$ mobile communication. With the development of science and technology of artificial intelligence, the general remote ground station with database servers confirm the mountain tunnel entrance to search. Even without the help of remote ground station staff manual control, UGV can automatic scan the environment.Then UGV confirms the mountain tunnel entrance with image processing technology. Then UGV drives into the tunnel to execute the searching task.

The sonar device on the UGV is like submarine sonar in underwater target detection. The sonar in the internal of the mountain tunnel performs "near-super far-near" data changes. When in the internal of the mountain tunnel, the sonar can help the UGV to determine the required search direction. However when UGV chooses the mountain tunnel entrance around the landing suites, the sonar can't replace the cameras carried on the UGV to find the entrance. As two parallel boulders, or two trees will perform the same sonar data, and sonar will mistakenly confirm the mountain tunnel entrance location. UGV must be equipped with image sensors to determine the mountain tunnel entrance location by image feature extraction of the mountain tunnel entrance. UGV then drive into the tunnel to search the potential targets.

\section{Image feature extraction for the mountain tunnel entrance}

The UGV turns to switch direction to scan the surrounding environment while the image sensor takes pictures to extract the characters of the mountain tunnel entrance.

Because the mountain tunnel is usually linear in the mountain to save costs in the construction periods, and UGV don't need to plan special routs. If UGV crashes onto the wall losing direction, UGV can turn left or right to find the correct direction again with the help of sonar carried on the UGV through the remote manual control from ground stations.

Excellent remote controlled UGV may not on duty at the stations for the emergency rescue task. To reduce the remote operator's fatigue and workload, UGV that search in the complex environment does not need the manual operation and it can by itself scan for mountain tunnel entrance and plan the path. Through the sonar carried on the UGV, UGV will choose the right direction to drive without crashing on to wall. UGV can scan the environment ahead with the image sensor carried on the UGV to confirm the potential targets in the tunnel.

\section{Hardware structure and implementation of the image characteristics of tunnel entrance}

\section{A. Extracting the contour of the image characteristics from the image sensor data}

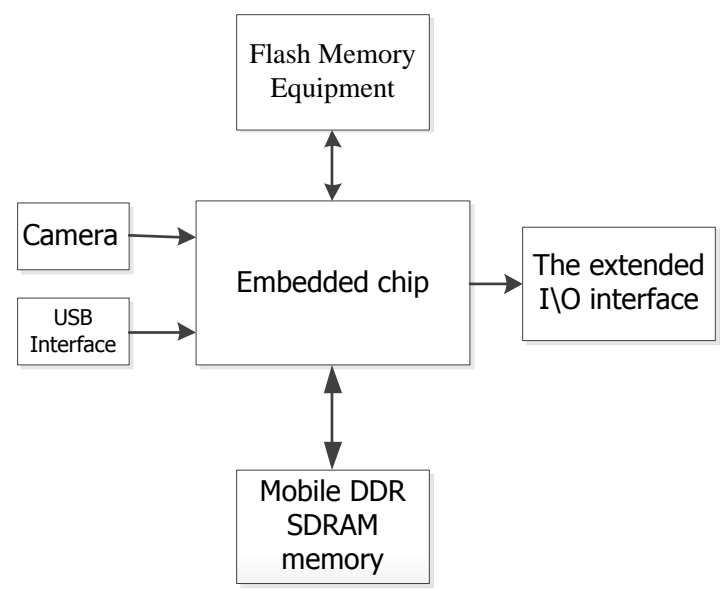

Fig. 1 The hardware structure diagram of image sensor carried on UGV

The USB camera is hidden in the body of UGV to prevent damage from collision and vibration. A kind of embedded operation system is carried on the UGV, too. By calling the camera driver interface function procedures can UGV realize the image acquisition mission about the potential targets in the internal of the mountain. The image acquisition and feature extraction process are shown in Fig. 2.

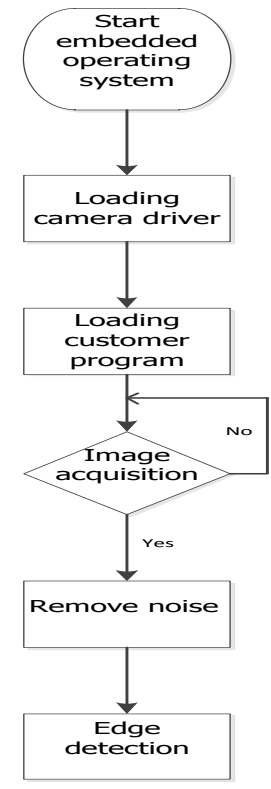

Fig. 2 Flow chart of image feature extraction 
The following expresses the process of UGV image sensor gets image data after the UGV landing around the mountain tunnel entrance. Firstly Function capInitCamera is called to initialize the image sensor on the UGV. Then by calling the Function capSetVideoFormat, the image sensor is set at an appropriate pixel video format and size. Finally the image sensor is launched by calling the Function capStartCamera. When UGV searches the character of mountain tunnel entrance, a frame of image is obtained by calling the Function capGrabFrame, and the image data is stored with the JPG format in the cache of information processing system. When UGV has confirmed the mountain tunnel entrance direction, it will drive into the tunnel to search targets. When UGV has received all of the information about the internal of the mountain tunnel, then the vehicle image sensor is stoped by calling the Function capStopCamera to close the vehicle image sensor by calling the Function capCloseCamera to save energy. Image acquisition system of the actual object is shown in Fig. 3.

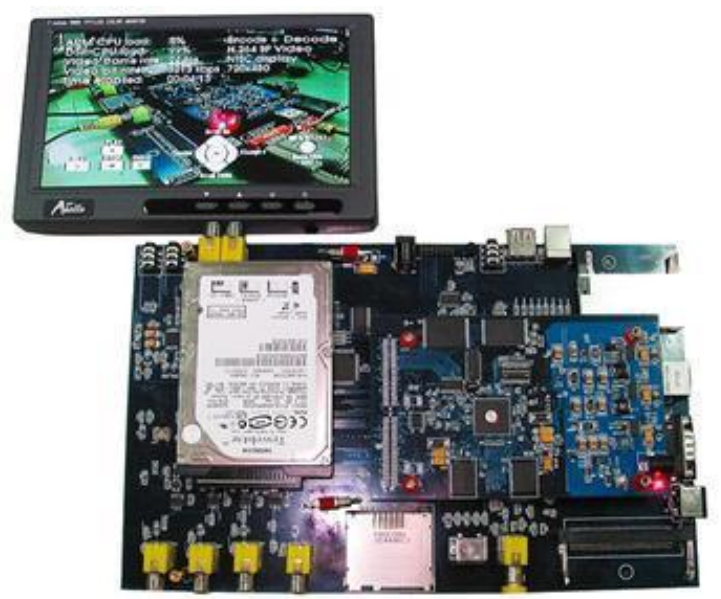

Fig. 3 Image acquisition system of the actual object

\section{B. Recognition feature extraction of mountain tunnel entrance}

In order to confirm the orientation information of tunnel entrance in the landing area of UGV, first of all the image edge and contour information of mountain tunnel entrance should be extracted. Although there are many algorithms for digital image processing of computer image contour feature, they can not simultaneously satisfy the requirements of realtime and miniaturization of unmanned vehicle search task. This paper studies a new digital image processing algorithms, written in MATLAB real-time image contour feature extraction of small procedures.

After repeated testing to ensure the calculation speed of the system is fast enough for real-time image processing quality, the on-board image sensor carried on UGV should apply $350 * 262$ pixel JPG format stored in SSD drive. Because of the complexity of environment in the search task, the image sensors should take more pictures, and SSD can perform more storage than other devices, as well as SSD has the character of uneasy to corrupt. $3 \mathrm{G}$ wireless communication may be interrupted when UGV driving in the internal of the mountain.
As a result, UGV drives to the open outdoor environment to upload the image data to the remote servers in ground station through the network of 3G SIM card.

When image sensor captures new images, firstly transforms the images into gray scale images with noise reduction to reduce the effect of complex environment. If UGV uploads the image data to the remote server through $3 \mathrm{G}$ network to process, the noise from the $3 \mathrm{G}$ network transmission should also be considered. Noise reduction methods commonly used are Mean filter, Laplace filter, Median filter method. By comparison, calculation of Median filter is simple and fast. Therefore Median filter is the most suitable methods for real-time requirements of continuous work of the noise reduction with remote control.

With the Median filtering method, at first $\mathrm{N}^{*} \mathrm{~N}$ scale size of the window ( $\mathrm{N}$ is odd) is selected. Secondly the values are sorted in the range of gray pixel size of $\mathrm{N}^{*} \mathrm{~N}$ in the image window. Thirdly the middle value of the pixel window center is taken as the gray value. In order to save the image and edge characteristics of mountain tunnel entrance with noise reduction, this paper adopts the $3 * 3$ median filter.

\section{Edge detection of image with contour extraction}

The outline of the image is the gray of pixel rapidly changing part in the neighborhood. With the construction of a variety of edge detection operator, adjacent pixels are changed. Commonly used edge detection operators are Sobel operator, Prewitt operator, Canny operator. Because the Canny operator edge detection performs the best quality, and performs the merit of fast processing speed [2]. It is suitable for the image processing with real-time requirement for searching task. This paper applies the Canny operator to get edge detection.

This paper applies the Otsu method to determine the threshold. At first the image is changed into one-dimensional histogram, then maximum value of variance between the background and objectives is selected as threshold. The edge contour tunnel entrance may exist in the image as the target, the remainder as background. Because of unmanned vehicle constantly turning to change direction to adjust for image sensors, unmanned vehicle observed the scene ahead. In many cases with a picture alone mountain tunnel entrance that unmanned vehicle will travel into will be determined.

Dispersion deals with between the statistical variance mountain tunnel entrance features of the target and the rest of the background scenery. As the variance of the numerical value is greater, the difference between outline and background is bigger. When part of mountain tunnel entrance with features as the target is judged wrong and divided into background, or vice verse, background is judged wrong as potential target, the variance value gets smaller. Therefore Variance between class $\sigma_{B}^{2}$ is maximum between the background and the mountain tunnel entrance contour, the error identification of target, background is minimized, and as a result the best threshold $t^{*}$ is found. The algorithm can correct the error of mistaken potential target as background. 
As a result it is very suitable for unmanned vehicle scanning the environment to confirm the direction of mountain tunnel entrance. Then unmanned vehicle drives into the internal of mountain tunnel to take the searching task.

The process of calculation for threshold is decomposed as follows. At first the pixels of just collected environment image are divided into $1,2,---$, L levels. For different gray level i, $f_{i}$ stands for the number of pixels. The total number of pixels in the image is defined as N. Obviously,

$N=f_{1}+f_{2}+\cdots+f_{L}=\sum_{i=1}^{L} f_{i}$. If $P_{i}$ stands for the

probability of the pixel in an image gray level $i$, then

$P_{i}=\frac{f_{i}}{N_{i}}$

$P_{i} \geq 0, \sum_{i=1}^{L} P_{i}=1$

. According to the threshold $\mathrm{t}$, the gray scale of the image can be divided into two categories. One is the gray level that is less than or equal to the threshold $t$, written as $C_{0}=\{1,2, \cdots, t\}$. The other is the gray level that is greater than threshold value $\mathrm{t}$, written as $C_{1}=\{t+1, t+2, \cdots, L\}$. The probabilities of two kinds of image gray level are expressed as

$$
\begin{aligned}
& \omega_{0}=P_{t}\left(C_{0}\right)=\sum_{i=1}^{t} P_{i}=\omega(t) \\
& \omega_{1}=P_{r}\left(C_{1}\right)=\sum_{i=t+1}^{L} P_{i}=1-\omega(t)
\end{aligned}
$$

Two kinds of gray value is expressed as

$$
\begin{aligned}
u_{0}=\sum_{i=1}^{t} i P_{r}\left(i \mid C_{0}\right)=\sum_{i=1}^{t} \frac{i P_{i}}{\omega_{0}}=\frac{u(t)}{\omega(t)} \\
u_{1}=\sum_{i=t+1}^{L} i P_{r}\left(i \mid C_{1}\right)=\sum_{i=t+1}^{L} \frac{i P_{i}}{\omega_{1}}=\frac{u_{L}-u(t)}{1-\omega(t)}
\end{aligned}
$$

in which

$$
u_{L}=u(L)=\sum_{i=1}^{L} i P_{i} \quad u(t)=\sum_{i=1}^{t} i P_{i} \quad \omega(t)=\sum_{i=1}^{t} P_{i}
$$

The sum of the two gray levels of probability is 1 for any threshold value t. It is easy to get $\omega_{0}+\omega_{1}=1$ and $\omega_{0} u_{0}+\omega_{1} u_{1}=u_{L}$. Variance between class $\sigma_{B}^{2}$ is used to evaluate the related decision function, written as

$$
\sigma_{B}^{2}=\omega_{0}\left(u_{0}-u_{L}\right)^{2}+\omega_{1}\left(u_{1}-u_{L}\right)^{2}
$$

Equation (1) and (2) are substituted in equation (3), then we get

$$
\sigma_{B}^{2}(t)=\frac{\left[u_{L} \omega(t)-u(t)\right]^{2}}{\omega(t)[1-\omega(t)]}
$$

Finally, the optimal threshold $t^{*}$ can be expressed as the corresponding threshold value $\mathrm{t}$ when the Variance between class $\sigma_{B}^{2}(\mathrm{t})$ gets the maximum value, which is written as

$$
\sigma_{B}^{2}\left(t^{*}\right)=\max \left\{\sigma_{B}^{2}(t)\right\}
$$

With the most appropriate threshold $t^{*}$ by the method described above, the image pixel gray value is transformed to 0 when it's less than or equal to the threshold value $t^{*}$. The image pixel gray value is transformed to 255 when it's more than the threshold value $t^{*}$ [3]. As a result, the two values of the images are realized, and image edge detection is achieved.

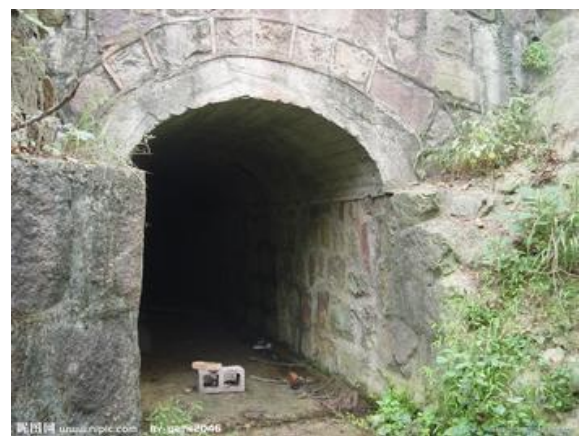

(a) Images from sensor carried by the unmanned vehicle

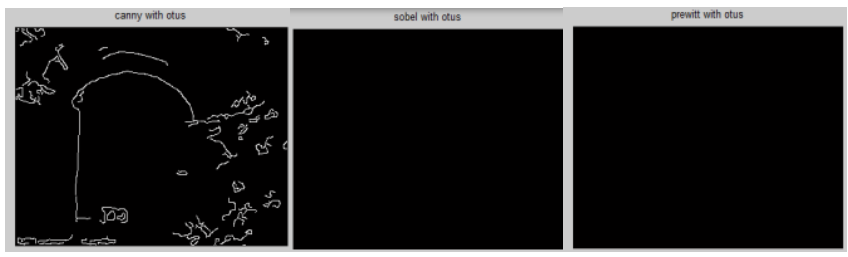

(b) Canny operator is better than Sobel and Prewitt operators when Otsu method is used to determine the gray level threshold.
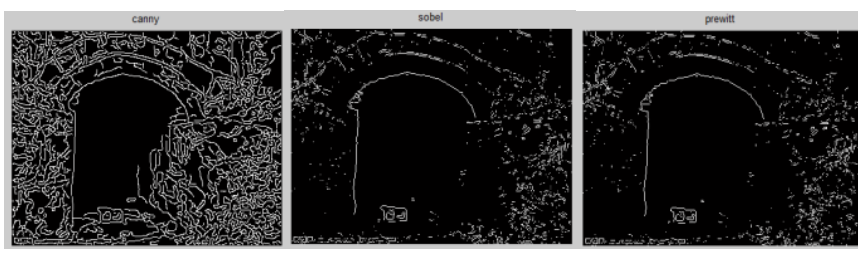

(c) Canny, Sobel and Prewitt operators are directly used for image contour extraction without the threshold determined by Otsu method. The noise is too high for the following treatment with machine vision technology .

Fig. 4 Extraction image features

It is showed that through manual design the threshold value of three operators, the threshold value determined by Otsu including between class variance maximum method, is higher than that can be used in Sobel and Prewitt operators. 
Sobel and Prewitt operators can't apply certain threshold value determined by otsu. Sobel and Prewitt operators have to be set threshold value that is lower than the calculation Otsu threshold to extract image contour.

The threshold determined by Otsu method is respectively put into the Canny, Sobel and Prewitt operators. Only Canny operator performs well in image contour extraction. Sobel and Prewitt operators do not extract the contour of the image. Through the manual design the operator's threshold, it shows that the threshold value determined by Otsu method with between class variance maximum is larger than that can be used in Sobel and Prewitt operators. Sobel operator and Prewitt operator with threshold value determined by Otsu method are not as good as Canny operator. The processing speed is fast enough for real-time search task. The contour is precision with small amount of calculation.

The experimental results show that with the image sensor carried by the unmanned vehicle, the Canny operator is used for the edge detection to determine the contour extraction of image. The Otsu method is used to determine the gray level threshold. The unmanned vehicle can drive in the direction of tunnel entrance without manual operator from remote ground station. The recognition for mountain tunnel entrance feature in complex environment, Canny operator is better than Sobel and Prewitt operators when Otsu method is used to determine the gray level threshold[4].

\section{Conclusion}

UAV needs UGV searching the areas, such as tunnel inside the mountain. The technology of UGV extraction image features of mountain tunnel portal is very important. The threshold determined by Otsu method is respectively put into the Canny, Sobel and Prewitt operators. Through experiment it is verified that Canny operator is better than Sobel and Prewitt operators when Otsu method is used to determine the gray level threshold.

\section{Acknowledgment}

This work was performed in the projects of both the National High Technology Research and Development Program of China (2011AA01A204) and the National Natural Science Foundation of China (91118002).

\section{References}

[1] Ulrik Franke, Waldo Rocha Flores, and Pontus Johnson, "Enterprise Architecture Dependency Analysis using Fault Trees and Bayesian Networks"

[2] Zhao Wencang. "Study of medical image segmentation algorithm based multi-wavelet analysis", 2008 IEEE International Conference on Automation and Logistics, 09/2008

[3] Jiang Zhu. "Obstacle detection and recognition in natural terrain for field mobile robot navigation", 2010 8th World Congress on Intelligent Control and Automation, 07/2010

[4] Feng-ying Cui. "Edge feature extraction based on digital image processing techniques", 2008 IEEE International Conference on Automation and Logistics, 09/2008 